\title{
Biological activity of green-synthesized silver nanoparticles depends on the applied natural extracts: a comprehensive study
}

This article was published in the following Dove Press journal:

International Journal of Nanomedicine

27 January 2017

Number of times this article has been viewed

\author{
Andrea Rónavári ${ }^{1, *}$ \\ Dávid Kovács ${ }^{2, *}$ \\ Nóra lgaz ${ }^{2, *}$ \\ Csaba Vágvölgyi ${ }^{3}$ \\ Imre Miklós Boros ${ }^{2,4}$ \\ Zoltán Kónya ${ }^{1,5}$ \\ Ilona Pfeiffer ${ }^{3}$ \\ Mónika Kiricsi \\ 'Department of Applied and \\ Environmental Chemistry, \\ ${ }^{2}$ Department of Biochemistry and \\ Molecular Biology, ${ }^{3}$ Department of \\ Microbiology, University of Szeged, \\ ${ }^{4}$ Institute of Biochemistry, Biological \\ Research Center of the Hungarian \\ Academy of Sciences, ${ }^{5}$ MTA-SZTE \\ Reaction Kinetics and Surface \\ Chemistry Research Group, Szeged, \\ Hungary \\ *These authors contributed equally \\ to this work
}

\begin{abstract}
Due to obvious disadvantages of the classical chemical methods, green synthesis of metallic nanoparticles has attracted tremendous attention in recent years. Numerous environmentally benign synthesis methods have been developed yielding nanoparticles via low-cost, eco-friendly, and simple approaches. In this study, our aim was to determine the suitability of coffee and green tea extracts in green synthesis of silver nanoparticles as well as to compare the performance of the obtained materials in different biological systems. We successfully produced silver nanoparticles (C-AgNP and GT-AgNP) using coffee and green tea extracts; moreover, based on our comprehensive screening, we delineated major differences in the biological activity of C-AgNPs and GT-AgNPs. Our results indicate that although GT-AgNPs exhibited excellent antimicrobial activity against all the examined microbial pathogens, these particles were also highly toxic to mammalian cells, which limits their potential applications. On the contrary, C-AgNPs manifested substantial inhibitory action on the tested microbes but were nontoxic to human and mouse cells, indicating an outstanding capacity to discriminate between potential pathogens and mammalian cells. These results clearly show that the various green materials used for stabilization and for reduction of metal ions have a defining role in determining and fine-tuning the biological activity of the obtained nanoparticles.
\end{abstract}

Keywords: green synthesis, silver nanoparticles, antimicrobial activity, toxicity

\section{Introduction}

Due to the widespread applications of nanomaterials in various fields of industry, technology, as well as medicine, the global demand for nanomaterials is growing exponentially. In fact, owing to the commercial utilization of silver nanoparticles (AgNPs) in optics, electronics, catalysis, in household items, and in a broad range of medical applications, the yearly increase in AgNP production was estimated to be hundreds of tons worldwide. ${ }^{1}$ Although photochemical reactions, ${ }^{2}$ thermal, ${ }^{3}$ radiation/sono-chemical, ${ }^{4}$ and microwave-assisted methods ${ }^{5}$ can be used successfully for efficient AgNP synthesis, the most general approaches are chemical reduction ${ }^{6}$ or electrochemical processes, ${ }^{7}$ which often involve the use of toxic chemicals causing harmful effects on the environment and human health. ${ }^{89}$ As an example, in bottom-up chemical preparation methods yielding stable colloidal AgNPs of desired sizes within the nanometer scale, sodium borohydride is frequently used as a reductant. ${ }^{10} \mathrm{NaBH}_{4}$, apart from being a fairly expensive chemical, can also generate oxidized boron species during the synthesis process, which cannot be easily separated from the nanomaterial product. ${ }^{11}$ Such undesired chemical entities can severely limit the application possibilities and the biocompatibility of the produced nanomaterials. Therefore, the utilization
Correspondence: Mónika Kiricsi Molecular Biology, University of Szeged, Tel +3662544887

Fax +36 62544887

Email kiricsim@bio.u-szeged.hu

Ilona Pfeiffer

Department of Microbiology, University of Szeged, Középfasor 52, H-6726

Szeged, Hungary

Tel +3662544517

$\mathrm{Fax}+3662544823$

Email pfeiffer@bio.u-szeged.hu 
of nanomaterials has to be accompanied with innovative, low-cost, eco-friendly production processes, which reduce the use of unsafe chemicals and minimize the generation of hazardous wastes. ${ }^{10,12}$

Green synthesis strategies of metal nanoparticles have recently gathered grounds, providing safer alternatives to conventional chemical methods and a low environmental footprint. Such approaches utilize mild experimental conditions (ambient temperature and pressure), require careful considerations in selecting nontoxic, environmentally benign solvents, reducing agents as well as capping materials ${ }^{11,12}$ and, therefore, frequently involve either living organisms, such as bacteria ${ }^{13}$ or fungi. ${ }^{14}$ Plants or natural extracts ${ }^{15}$ obtained from plant leaf, root, seed, and stem are also frequently applied for green nanomaterial production, as these extracts contain various polysaccharides, proteins, vitamins, or alkaloids, which are generally nontoxic, biodegradable, and can act both as reducing and capping agents, thereby promoting the formation and inhibiting the agglomeration of nanoparticles..$^{9,11,12,15-18}$ Typically, such a synthesis is very straightforward, the metal salt solution needs to be mixed with the natural product extract, and nanoparticles form spontaneously within a few minutes up to 1 day. ${ }^{19}$ One of the first approaches along this line by Shankar et al reported the rapid synthesis of metallic AgNPs at high concentrations by treating aqueous solutions of silver nitrate with neem (Azadirachta indica) leaf broth. ${ }^{20}$ These authors emphasized that the major advantage of this method was the rate of metal ion reduction by the plant extract, which proved to be much faster than those observed previously using microorganisms. ${ }^{20}$ Ever since, AgNPs of different shapes and sizes were obtained using various plant extracts, such as Aloe vera, ${ }^{21}$ crop plants, ${ }^{12}$ Citrus limon, ${ }^{22}$ Capsicum annuum, ${ }^{23}$ just naming a few, as reducing agents. ${ }^{24}$ Active ingredients of coffee (C) or tea such as polyphenols or flavonoids can also be applied to render production routes green and cost-effective. ${ }^{25}$ Hydroalcoholic extract of dried roasted Coffea arabica seed, ${ }^{25}$ green tea (GT) and black tea $^{26,27}$ extracts have already been used for the biosynthesis of AgNPs, where the phenolic acid-type caffeine and theophylline were responsible for the stabilization of the obtained nanomaterials.

Although the synthesis of nanoparticles using biological methodologies has received increasing attention in the last decade, only a few studies reported reliable data on the biological activities of the obtained green-synthesized nanomaterials, highlighting the differences of nanoparticle action in various biological host systems. Our approach is novel due to the fact that we aimed to compare above all the biological performance of two types of silver colloids obtained by totally green chemical reduction methods using GT and C extracts. The green-synthesized C-AgNP and GT-AgNPs were characterized, and in the frame of comprehensive screening, nanoparticles were tested against various microbes and mammalian cells to delineate major differences between the biological efficacy of C-AgNPs and GT-AgNPs.

\section{Materials and methods Preparation of plant extracts}

To prepare the GT extract, 2 g of dry GT leaves (R. Twining and Company Limited, London, England) was soaked in $100 \mathrm{~mL}$ deionized water and the solution was heated to $80^{\circ} \mathrm{C}$ for $20 \mathrm{~min}$, thereafter the extract was cooled, vacuum-filtered, and this filtrate was further used as a reducing agent and also as a stabilizer of the as-synthesized AgNPs. A similar process was applied for the $C$ extract except that the purchased coffee (Tchibo Family) was boiled for $5 \mathrm{~min}$.

\section{Synthesis of AgNPs}

Silver nanoparticles (GT-AgNP and C-AgNP) were synthesized by adding the corresponding extracts to $0.1 \mathrm{M}$ aqueous silver nitrate $\left(\mathrm{AgNO}_{3}, \geq 99.0 \%\right.$; Sigma-Aldrich) solution in a 1:1 volume ratio at room temperature, $\mathrm{pH} 7$, by constant stirring for $24 \mathrm{~h}$. Preliminary optimization experiments indicated that the application of $1: 1$ green extract $/ \mathrm{AgNO}_{3}$ ratio is required to achieve particles with spherical morphology. The obtained disperse system was then purified by repeated centrifugation at $1,730 \times g$ for $5 \mathrm{~min}$. The supernatant was transferred to a clean dry beaker for further settlement of particles, and repeated cycles of centrifugation were carried out to further purify AgNPs. The final colloid samples were stored at $4^{\circ} \mathrm{C}$.

\section{Characterization of nanoparticles}

The morphological characteristics of AgNPs were examined by transmission electron microscopy (TEM) using a FEI Tecnai $\mathrm{G}^{2} 20 \times$ microscope at an acceleration voltage of $200 \mathrm{kV}$. The crystal structures were analyzed by X-ray powder diffraction (XRD). The scans were performed with a Rigaku MiniFlex II powder diffractometer using $\mathrm{Cu} \mathrm{K} \alpha$ radiation. A scanning rate of $2^{\circ} \mathrm{min}^{-1}$ in the $5^{\circ}-80^{\circ} 2 \theta$ range was used. The particle size distribution of the samples was assessed by dynamic light scattering (DLS) analysis using a Zetasizer Nano Instrument (Malvern, Worcestershire, UK). The optical properties of nanoparticles were studied by 
spectral analysis. The absorbance spectra of nanoparticles were recorded within the range from 350 to $900 \mathrm{~nm}$ using an Ocean Optics 355 DH-2000-BAL UV-VIS spectrophotometer and a $10-\mathrm{mm}$ path length quartz cuvette.

Fourier transform infrared spectroscopy (FT-IR) studies were carried out using a Bruker Vertex 70 spectrophotometer. The C-AgNP and the GT-AgNP samples were washed thoroughly three times with distilled water prior to FT-IR experiments to remove organic compounds not bound to nanoparticles surfaces. The obtained samples were prepared using the $\mathrm{KBr}$ pellet technique and were analyzed to check the presence of biofunctional moieties of $\mathrm{C}$ and GT extracts and the surface chemistry of the reduced silver samples. The FT-IR spectra were collected at a spatial resolution of $4 \mathrm{~cm}^{-1}$ in the transmission mode, between 4,000 and $450 \mathrm{~cm}^{-1}$, respectively.

To determine the amount of silver ion released by green-synthesized AgNPs, inductively coupled plasma mass spectroscopy (ICP-MS, Agilent 7700x) was applied. The nanoparticles were dissolved and incubated for $24 \mathrm{~h}$ in culture medium (Dulbecco's Modified Eagle's Medium [DMEM containing $1 \mathrm{~g} / \mathrm{L}$ glucose] supplemented with $10 \%$ fetal bovine serum [FBS], 2 mM L-glutamine, 0.01\% streptomycin, and $0.005 \%$ ampicillin) in a $17.5 \mathrm{mg} / \mathrm{mL}$ concentration. The following day the solutions were centrifuged for $20 \mathrm{~min}$ at $10,285 \times \mathrm{g}$, and the supernatants of the respective samples were prepared for ICP-MS analysis by acid digesting using 1\% nitric acid (analytical quality cc. $\mathrm{HNO}_{3}$ in Milli-Q water).

\section{Cell culture}

Human cervical cancer HeLa cells and mouse NIH/3T3 noncancerous fibroblast cells were purchased from ATCC. HeLa cells were maintained in DMEM containing $1 \mathrm{~g} / \mathrm{L}$ glucose, supplemented with 10\% FBS, 2 mM L-glutamine, 0.01\% streptomycin, and $0.005 \%$ ampicillin. NIH/3T3 cells were grown on high glucose $(4.5 \mathrm{~g} / \mathrm{L})$ DMEM medium, containing 10\% FBS, 2 mM L-glutamine, 0.01\% streptomycin, and $0.005 \%$ ampicillin. Cells were cultured under standard conditions in a $37^{\circ} \mathrm{C}$ incubator at $5 \% \mathrm{CO}_{2}$ in $95 \%$ humidity.

\section{Cell proliferation and cytotoxicity assays}

To measure cell proliferation, MTT mitochondrial activity assay was performed: 5,000 HeLa cells/well and 2,000 $\mathrm{NIH} / 3 \mathrm{~T} 3$ cells/well were seeded into 96-well plates and treated with AgNPs in different concentrations on the following day. After $24 \mathrm{~h}$ treatments, cells were washed with PBS and incubated with culture medium containing $0.5 \mathrm{mg} / \mathrm{mL}$ MTT reagent (Sigma-Aldrich) for an hour at $37^{\circ} \mathrm{C}$. Formazan crystals were solubilized in dimethyl sulfoxide, and extinction was measured at $570 \mathrm{~nm}$ using a Synergy HTX plate reader. Absorption corresponding to the untreated control samples was considered as $100 \%$. MTT assays were performed at least three times using four independent biological replicates.

Cytotoxicity of the green-synthesized nanoparticles was assessed by crystal violet staining. Cells were seeded into 24-well plates and were left to grow until they reached confluence. Then, cell layers were treated with nanoparticles for $24 \mathrm{~h}$. After each treatment, cells were washed three times with PBS and fixed using methanol/acetone (70:30) mixture. Fixed cells were then stained using $0.5 \%$ crystal violet, washed with distilled water, and then air-dried. Plates were photographed and crystal violet was solubilized using $400 \mu \mathrm{L}$ of $10 \%$ acetic acid. A volume of $100 \mu \mathrm{L}$ solution from each well was transferred to 96-well plates, and then, absorbance was determined at $590 \mathrm{~nm}$ using a Synergy HTX microplate reader.

\section{Screening of antibacterial and antifungal activity}

GT-AgNPs and C-AgNPs were tested for antimicrobial activity against Escherichia coli SZMC 0582, Pseudomonas aeruginosa SZMC 0568, Micrococcus luteus SZMC 0264, Bacillus cereus var. mycoides SZMC 0042, Cryptococcus neoformans IFM 5844, Candida albicans ATCC 10231, Candida parapsilosis CBS 604, and Saccharomyces cerevisiae SZMC 20733 by agar diffusion method as described previously. ${ }^{28}$ The growth medium for cultivation of yeasts was yeast extract-peptone-D-glucose (YPD) (1\% peptone, $1 \%$ glucose, $0.5 \%$ yeast extract, and $2 \%$ agar), whereas for bacteria nutrient agar supplemented with glucose $(0.1 \%$ meat extract, $0.5 \%$ peptone, $1 \%$ glucose, $0.2 \%$ yeast extract, and $2 \%$ agar) was used. AgNP suspensions were prepared in $17.5 \mathrm{mg} / \mathrm{mL}$ final concentrations and $5 \mu \mathrm{L}$ of each was loaded directly onto the surface of agar plates seeded with the test strains. The plates were incubated at $30^{\circ} \mathrm{C}$, and the inhibition zones were determined after $24 \mathrm{~h}$.

The colony-forming units of $C r$. neoformans and E. coli were established to quantify the antimicrobial effectiveness of AgNPs prepared by C or GT extracts. Briefly, $4 \times 10^{6}$ yeast or $2 \times 10^{7}$ bacterial cells were exposed to C-AgNPs or to GT-AgNPs at concentrations of $17.5 ; 70 ; 175 ; 350$; and $1,750 \mu \mathrm{g} / \mathrm{mL}$ for $24 \mathrm{~h}$. After the incubation time, cells were washed out from the suspension and then were suspended in sterile water. A dilution series was prepared, and $25 \mu \mathrm{L}$ aliquots of each dilution were spread onto YPD or nutrient agar medium in triplicates. Plates were incubated at $30^{\circ} \mathrm{C}$ 
for $48 \mathrm{~h}$, and the number of the colonies was counted. The experiments were carried out always in triplicates.

\section{Determination of yeast killer activity}

An overnight culture of the K1 killer toxin ${ }^{29}$ producing strain $S$. cerevisiae SZMC 20733 was diluted to a density of $1 \times 10^{6}$ cells $/ \mathrm{mL}$. $1 \mathrm{~mL}$ aliquots of the suspension were pelleted by centrifugation and resuspended in $1 \mathrm{~mL}$ of either $1.75 \mathrm{mg} / \mathrm{mL}$ C-AgNP or $17.5 \mu \mathrm{g} / \mathrm{mL}$ GT-AgNP solution. Samples were placed in a rotary shaker at $30^{\circ} \mathrm{C}$ for $24 \mathrm{~h}$. After incubation, cells were collected by centrifugation, washed, and suspended in $1 \mathrm{~mL}$ water. A volume of $50 \mu \mathrm{L}$ aliquots were plated on malt agar (3\% malt extract, $0.5 \%$ yeast extract, and $2 \%$ agar) and incubated at $30^{\circ} \mathrm{C}$ for 4 days. At least 50 single colonies were randomly selected and transferred to malt agar plates. After 2 days of incubation at $37^{\circ} \mathrm{C}$, colonies were replica plated onto the surface of a medium $(2 \%$ glucose, $2 \%$ peptone, $1 \%$ yeast extract, and $2 \%$ agar, buffered to pH 4.5 with citrate-phosphate buffer) previously inoculated with a turbid solution of $S$. cerevisiae SZMC 23464 killer sensitive strain.

Five colonies with altered killer phenotype were isolated from C-AgNP-treated plates. The colonies were inoculated into YPD broth and were cultivated for $24 \mathrm{~h}$ at $30^{\circ} \mathrm{C}$ with vigorous shaking. After the incubation time, $50 \mu \mathrm{L}$ aliquots were transferred into $2 \mathrm{~mL}$ fresh YPD broth and further incubated for $24 \mathrm{~h}$ at $30^{\circ} \mathrm{C}$ with vigorous shaking. The transfer of the cells into fresh YPD media was repeated over 7 days and, finally, their killer activity was determined by loading $10 \mu \mathrm{L}$ suspensions onto the surface of the above-mentioned medium inoculated with the killer sensitive strain.

For viral RNA isolation, cells from 3-day-old cultures of the isolates with altered phenotype and of the untreated control strain were washed and resuspended in PBS and disrupted in French-press at 20,000\%, and then cell debris was pelleted by centrifugation $\left(25,000 \times g, 4^{\circ} \mathrm{C}, 30 \mathrm{~min}\right)$. About $200 \mu \mathrm{L}$ of supernatant was digested with RNase A (SigmaAldrich) $\left(50 \mu \mathrm{g} / \mathrm{mL}, 30 \mathrm{~min}\right.$ at $\left.37^{\circ} \mathrm{C}\right)$, proteins were removed by phenol-chloroform extraction, and viral RNA from the supernatant was resolved by agarose gel electrophoresis.

\section{Results}

\section{Characterization of AgNPs}

AgNPs were visualized by TEM, which indicated that both the GT-AgNPs and the C-AgNPs have spherical morphology. According to TEM images, C-AgNPs were monodisperse, the particles were well separated from each other, however, were trapped in the matrix of the residual $\mathrm{C}$ extract. In contrast to C-AgNPs, GT-AgNPs were larger, minor polydispersity was observed, and the particles formed loose aggregates (Figure 1A). Based on image analysis, the average size of the particles proved to be $3.2 \pm 1.2 \mathrm{~nm}$ for C-AgNP and $12.7 \pm 5.8 \mathrm{~nm}$ for GT-AgNP, respectively.

XRD studies were performed to confirm the crystalline structure of the synthesized nanoparticles. The XRD pattern (Figure 1B) of both AgNPs showed four intense characteristic reflections at the $2 \theta$ angles of $38.2^{\circ}, 44.3^{\circ}, 64.4^{\circ}$, and $77.4^{\circ}$, respectively, corresponding to (111), (200), (220), and (311) planes of face-centered cubic lattice structure of metallic silver (JCPDS No. 87-0717)..$^{30}$

DLS measurements were carried out to determine hydrodynamic particle sizes (Figure 1C). The average size of C-AgNP was between 8 and $20 \mathrm{~nm}$, whereas the hydrodynamic size of GT-AgNPs was between 20 and $50 \mathrm{~nm}$. GT-AgNPs manifested an additional peak at around $400 \mathrm{~nm}$ indicating mild agglomeration.

UV-VIS spectra of AgNPs prepared by C and GT extracts are shown in Figure 1D. Peak maxima at $456 \mathrm{~nm}$ for GTAgNPs and at $469 \mathrm{~nm}$ for C-AgNPs characteristic to surface plasmon resonance also supported the formation of AgNPs.

The quality and the stability of the AgNP colloid solutions were frequently monitored during the timeframe of the biological experiments. AgNPs were stable, no aggregation was detected. Figure $1 \mathrm{C}$ and D and Figure S1 verify that no significant changes occurred in the prepared nanoparticle samples throughout the experimental period.

FT-IR analysis was performed on biological extracts and on the AgNP samples obtained by the application of these extracts to identify the functional groups of the chemical components of these samples (Figure 1E). All samples contain alcoholic, phenolic, and carboxylic groups, indicated by an intense broad band around $3,400 \mathrm{~cm}^{-1}$, corresponding primarily to $\mathrm{O}-\mathrm{H}$ stretching of hydroxyl groups, as well as to primary and secondary amines and amides denoted by N-H stretching vibrations. This band can be attributed to the nondissociatively adsorbed water molecules as well, the existence of the latter can be unambiguously proven by the presence of a band at 1,620 $\mathrm{cm}^{-1}$ due to the deformation vibration of the water molecules. The peak around $1,145 \mathrm{~cm}^{-1}$ is attributed to the $\mathrm{C}-\mathrm{N}$ stretching vibration of aromatic primary and secondary amines, and the bands at $900-600 \mathrm{~cm}^{-1}$ correspond to primary and secondary amines and amides ( $-\mathrm{NH}_{2}$ wagging). The presence of ketones, aldehydes, quinines, and esters could be indicated by the peaks between 1,700 and $1,600 \mathrm{~cm}^{-1}$, assigned to the $\mathrm{C}=\mathrm{O}$ vibration of carbonyl groups. The band at $2,920 \mathrm{~cm}^{-1}$ is attributed to $\mathrm{C}-\mathrm{H}$ stretching of alkanes. The peak at about $1,630 \mathrm{~cm}^{-1}$ is credited 
A
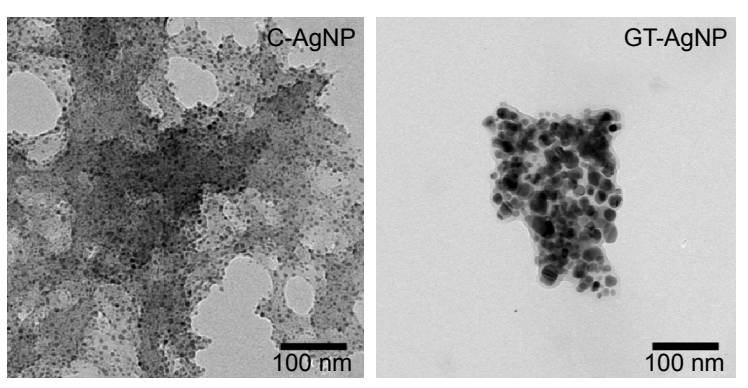

C

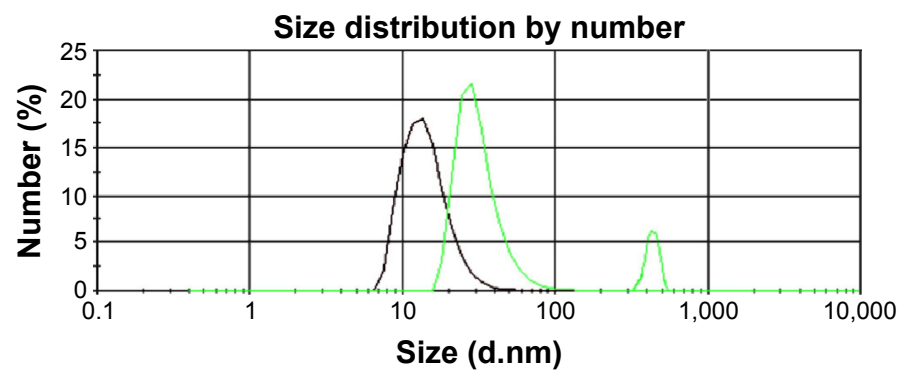

B

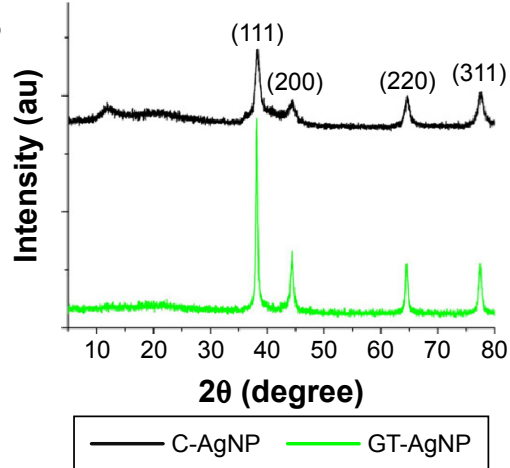

D

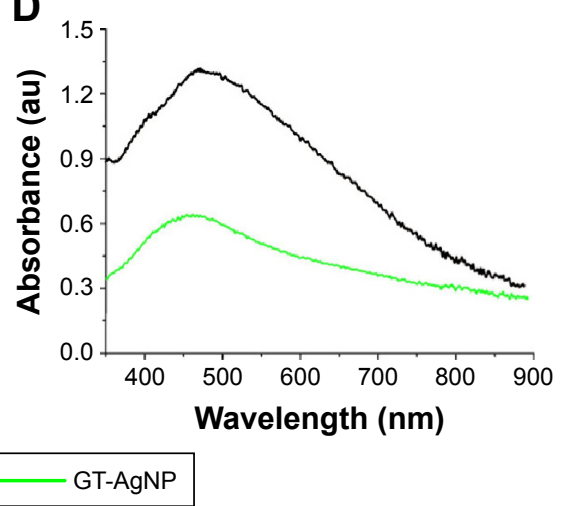

E

C-AgNP GT-AgNP

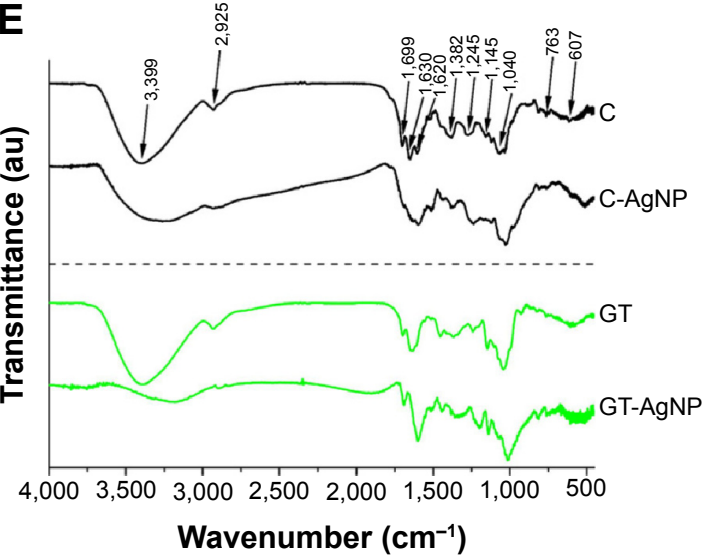

Figure I Physicochemical characterization of the obtained silver nanoparticles.

Notes: Representative transmission electron microscopic images (A), X-ray diffraction patterns (B), size distribution by dynamic light scattering (C), and ultraviolet-visible spectra (D) of silver nanoparticles synthesized by coffee (C-AgNP) and green tea extracts (GT-AgNP). (E) FT-IR spectra of coffee extract (C), GT, green-synthesized C-AgNPs and GT-AgNPs.

Abbreviations: C, coffee; FT-IR, Fourier transform infrared spectroscopy; GT, green tea; NP, nanoparticle.

to the $\mathrm{C}=\mathrm{C}$ vibration of aromatic structures, whereas the peak at $1,245 \mathrm{~cm}^{-1}$ is assigned to $\mathrm{C}-\mathrm{O}$ stretching of phenolic groups. Another broad band centered around $1,040 \mathrm{~cm}^{-1}$ is attributed to aromatic ethers and polysaccharides $\left(\mathrm{C}-\mathrm{O}-\mathrm{C}\right.$ stretch). ${ }^{31}$ Carbonyl groups suggested the presence of proteins, and $\mathrm{OH}$ functional groups indicated phenolic compounds in our $\mathrm{C}$ and GT extracts. Our FT-IR results are in good agreement with the literature data, that is, the surface of the AgNPs is covered by organic compounds derived from C and GT extracts (see the $900-1,800 \mathrm{~cm}^{-1}$ region).

\section{Toxicity on human cells}

We evaluated the antiproliferative propensity and the cytotoxicity of C-AgNP and GT-AgNPs using human cancerous HeLa and mouse noncancerous NIH/3T3 cells. MTT assays revealed that GT-AgNPs inhibited the proliferation of both cell types with a greater extent than C-AgNPs (Figure $2 \mathrm{~A}$ and $\mathrm{B}$ ). According to the calculated $\mathrm{IC}_{50}$ values, NIH/3T3 fibroblasts (GT-AgNP: $\sim 5 \mu \mathrm{g} / \mathrm{mL}$; C-AgNP: $272.14 \pm 0.09 \mu \mathrm{g} / \mathrm{mL}$ ) were more sensitive to AgNP exposures than cervical cancer HeLa cells (GT-AgNP: $14.26 \pm 0.05 \mu \mathrm{g} / \mathrm{mL}$; 
A

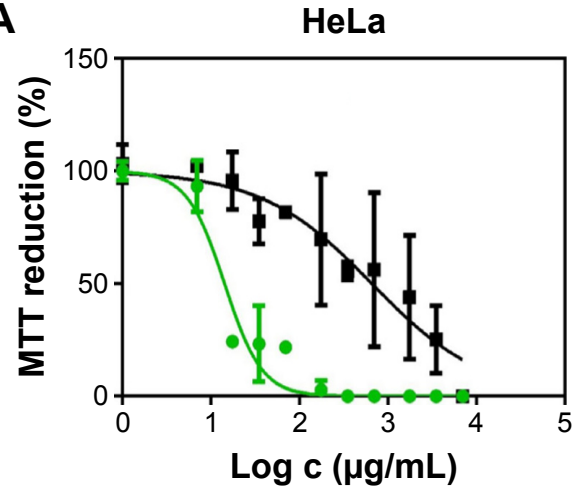

B

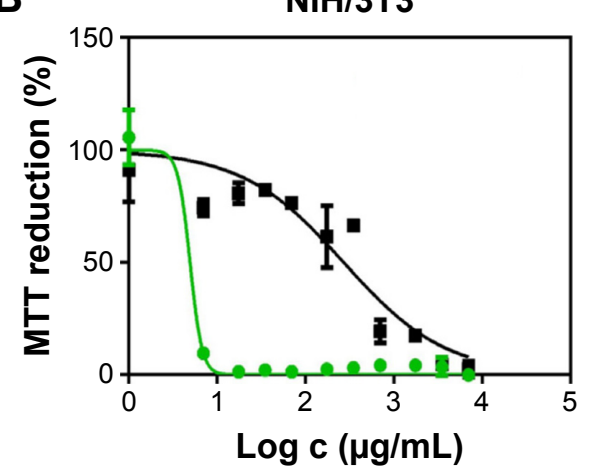

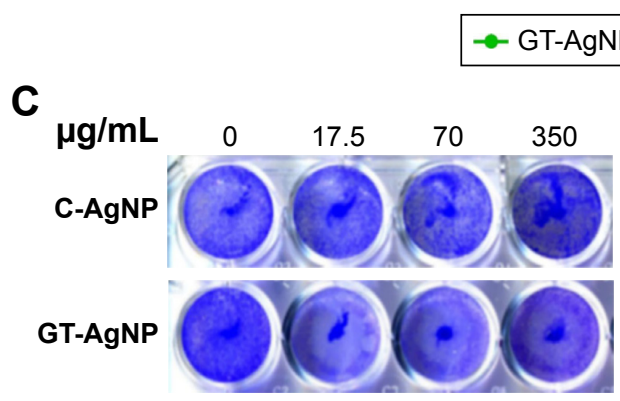

D

E

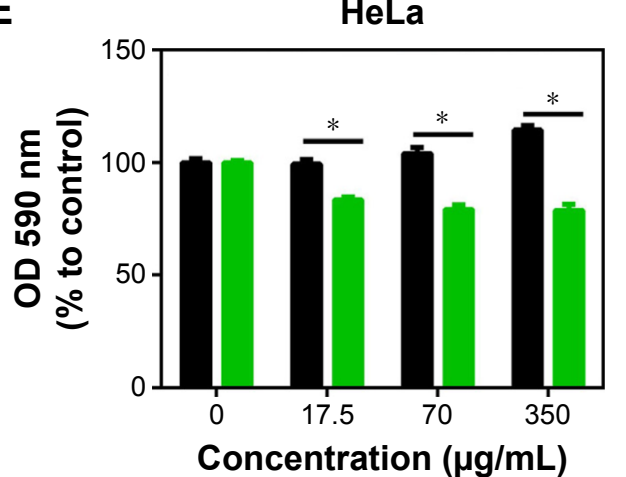

$\begin{array}{lllll}\boldsymbol{\mu g} / \mathbf{m L} & 0 & 17.5 & 70 & 350\end{array}$

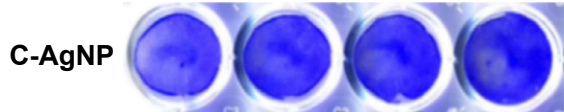

GT-AgNP

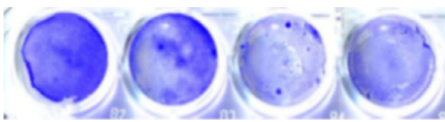

$\mathbf{F}$

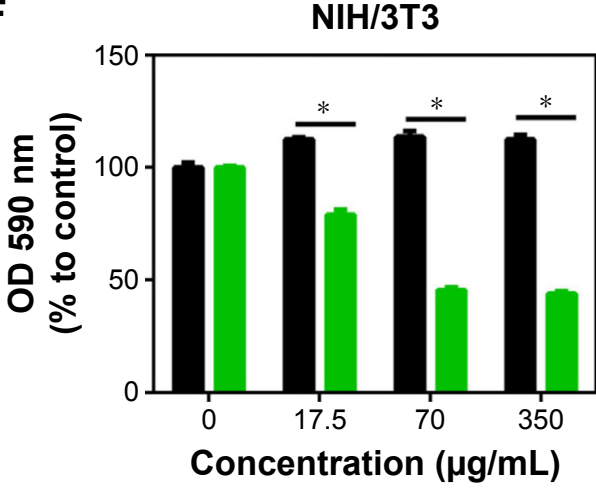

- C-AgNP GT-AgNP

Figure 2 Antiproliferative and cytotoxic effects of green-synthesized silver nanoparticles on mammalian cell lines.

Notes: C-AgNP and GT-AgNP treatments inhibit the proliferation of human HeLa (A) and mouse NIH/3T3 fibroblast cells (B). Cytotoxic effects of C-AgNP and GT-AgNP treatments were determined on HeLa (C) and NIH/3T3 (D) cells using crystal violet staining. Crystal violet was resolubilized and the absorbance of each sample was determined spectrophotometrically (E, F). *P $\leq 0.00$ I, two-way ANOVA.

Abbreviations: ANOVA, analysis of variance; C, coffee; GT, green tea; NP, nanoparticle; OD, optical density.

C-AgNP: $655.3 \pm 0.12 \mu \mathrm{g} / \mathrm{mL}$ ). Furthermore, in both cell types, GT-AgNPs exhibited $\sim 50$ times higher inhibition on cell proliferation than C-AgNPs (Figure 2A and B).

Toxicity of both AgNPs was analyzed by crystal violet staining method (Figure 2C-F). AgNPs synthesized by C extract were nontoxic to the applied human and mouse cells in the tested concentration range. On the contrary, GT-AgNPs proved to be significantly cytotoxic to both $\mathrm{HeLa}$ as well as to NIH/3T3 fibroblast cells; however, the latter cells were more sensitive to the toxic effects of GT-AgNP. These results indicate that GT-AgNPs exhibit stronger antiproliferative activity and cytotoxicity than C-AgNPs.

\section{Antibacterial and antifungal activity}

To determine whether the prepared AgNPs are effective against selected bacteria and fungi and to identify which of the two green-synthesized AgNPs exhibit better antimicrobial features, we tested the C-AgNPs and GT-AgNPs against B. cereus var. mycoides, M. luteus, E. coli, and $P$. aeruginosa in addition against nonpathogenic and pathogenic yeasts $S$. cerevisiae, C. parapsilosis, C. albicans, and $\mathrm{Cr}$. neoformans. Figure 3 shows that nanoparticles prepared either by $\mathrm{C}$ or GT extracts proved to be effective against all the examined bacterial strains (Figure 3A). Similar to bacteria, the tested pathogenic fungi, namely C. parapsilosis, 
A


C

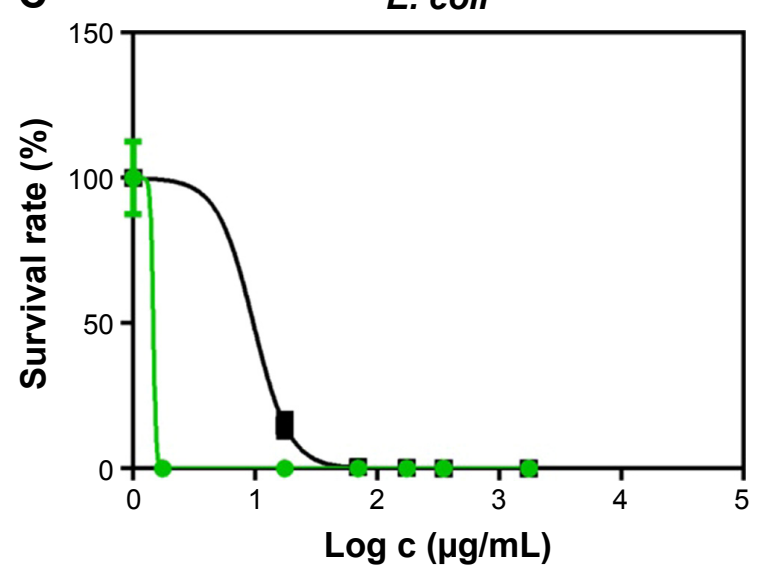

B
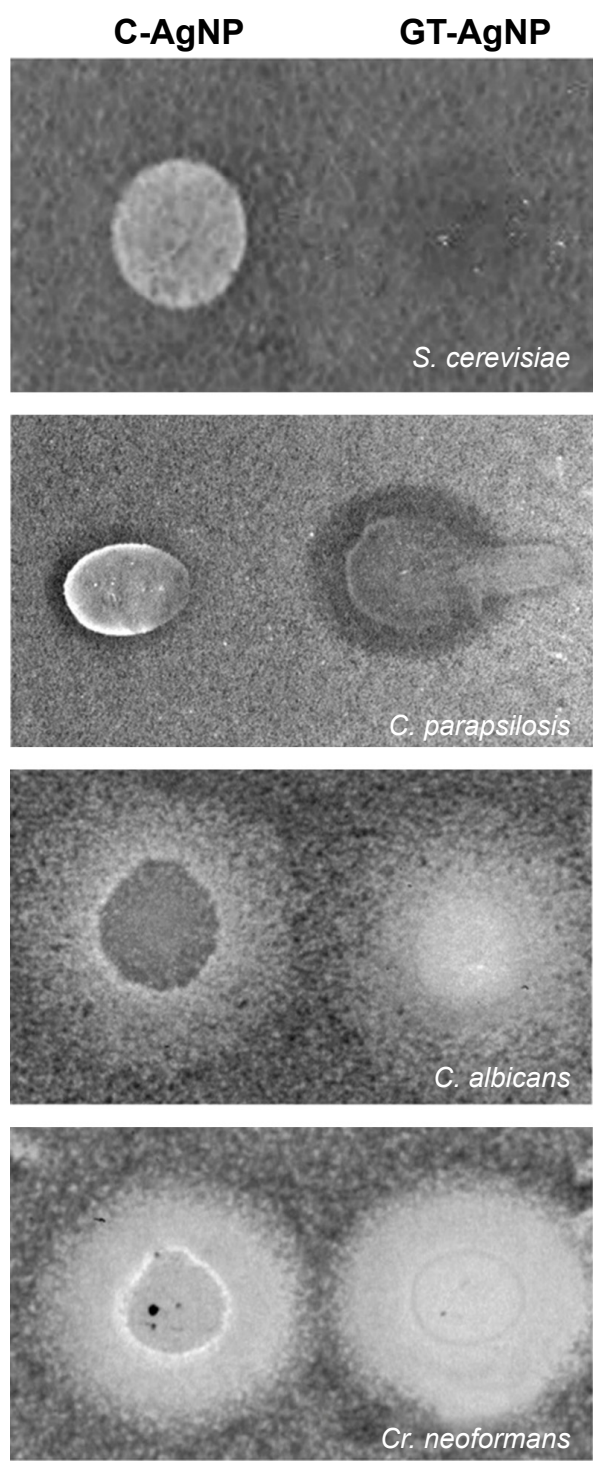

D

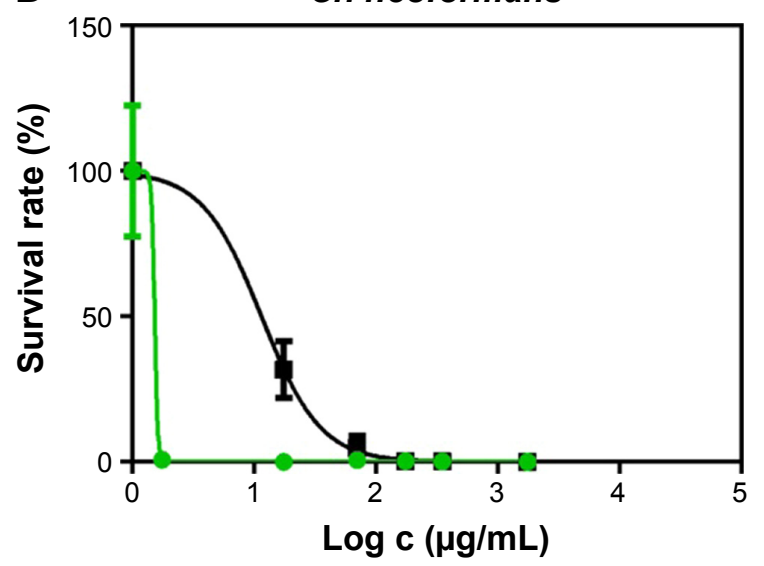

$\rightarrow-$ C-AgNP $\rightarrow$ GT-AgNP

Figure 3 Antimicrobial activities of green-synthesized silver nanoparticles.

Notes: Agar diffusion assay. Plates were inoculated with Bacillus cereus var. mycoides, Micrococcus luteus, Pseudomonas aeruginosa, and Escherichia coli strains (A) and with Saccharomyces cerevisiae, Candida parapsilosis, Candida albicans, and Cryptococcus neoformans (B). Survival rate of C-AgNP- and GT-AgNP-treated E. coli (C) and Cr. neoformans (D).

Abbreviations: C, coffee; GT, green tea; NP, nanoparticle. 
C. albicans, and Cr. neoformans, were also susceptible to both nanoparticles, as large inhibition zones could be recorded around these yeasts (Figure 3B; Table S1). The nonpathogenic $S$. cerevisiae was neither affected by C-AgNPs nor by GT-AgNPs in the applied concentration, as in this case no inhibition zones could be observed (Figure 3B).

As the agar diffusion method is not able to reveal quantitative differences in the effectiveness of C-AgNPs and GT-AgNPs, microbe survival rates were determined at different AgNP concentrations on E. coli and on Cr. neoformans strains. As it is demonstrated in Figure 3C and D, C-AgNPs killed E. coli and $\mathrm{Cr}$. neoformans cells in a dose-dependent manner; however, GT-AgNP treatments resulted in complete loss of viability even in the lowest tested concentration (Figure 3C and D).

\section{Antiviral activity}

The antiviral activity of C-AgNPs and GT-AgNPs was investigated using $S$. cerevisiae dsRNA viruses, which are responsible for the killer phenotype of the host strain. Using this model system, the possible influence of AgNPs on viral replication can be detected easily by observing the loss of killer phenotype of the host strain (Figure S2) and monitoring the presence or absence of the viral genome. Five strains with altered phenotype were isolated after C-AgNP treatment (Figure S2B, indicated by numbers 1-5), while GT-AgNP treatment resulted in three nonkiller strains (Figure S2C, indicated by arrows).

The stability of the altered phenotype was further examined in the strains originated from C-AgNP treatment. After transferring the strains day-to-day into fresh culture medium over 7 days ( $\sim 112$ generations), only one strain maintained the nonkiller phenotype (Figure $4 \mathrm{~A}-\# 2$ ), whereas two strains produced narrow inhibition zones (Figure 4A - \#1 and \#4) compared with the control (Figure 4A $[\mathrm{K}]$ ). Interestingly, viral genomes could be detected in all the five strains (Figure 4B), including the nonkiller one (Figure 4B - \#2), indicating that the loss of killer phenotype was not due to viral eradication.

\section{Release of silver ions}

To reveal the possible explanation behind the differences in biological activities between C-AgNP and GT-AgNP, we measured the concentration of silver released from nanoparticles after 1-day incubation period in cell culture medium by ICP-MS. C-AgNPs as well as GT-AgNPs were dissolved in serum containing DMEM medium in a final concentration of $17.5 \mathrm{mg} / \mathrm{mL}$, respectively. After $24 \mathrm{~h}$ incubation at $37^{\circ} \mathrm{C}$, supernatants were obtained by centrifugation and prepared for ICP-MS. According to these measurements, the concentration of dissolved silver was $0.59 \pm 0.02 \mathrm{mg} / \mathrm{mL}$ for C-AgNPs and $1.92 \pm 0.07 \mathrm{mg} / \mathrm{mL}$ for GT-AgNPs, which indicated that $\sim 3.5$ times more silver ions can be released from GT-AgNPs than from C-AgNPs. These results suggest that although $\mathrm{C}$-AgNPs are approximately four times smaller than GT-AgNPs, less $\mathrm{Ag}^{+}$can be dissolved from the surface of C-AgNPs, probably due to the stabilizing matrix surrounding the particles, which also accounts for their reduced toxicities compared with GT-AgNPs.

\section{Discussion}

The application of nontoxic, biocompatible agents renders green nanoparticle synthesis eco-friendly and cost-effective. Just as the awareness and the interest in green methodologies increases, so expands the scientific literature describing the various biological entities exploited for the green biosynthetic route of metal nanoparticles. Phototrophic eukaryotic organisms, like algae or plants, or their extracts, heterotrophic eukaryotic cell lines and numerous prokaryotes, as well as
A

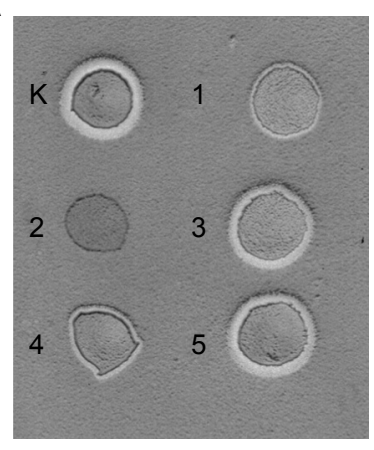

B

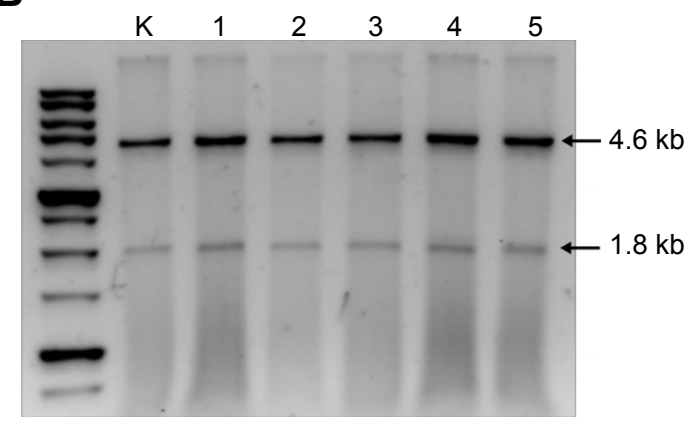

Figure 4 Antiviral effect of silver nanoparticles.

Notes: Killer phenotype of Saccharomyces cerevisiae SZMC 20733 (K) and C-AgNP-treated strains (I-5) after II2 generations (A). Viral RNA extracted from SZMC $20733(\mathrm{~K})$ and from C-AgNP-treated strains (I-5). Arrows indicate the presence of L-A (4.6 kb) and MI (I.8 kb) virus genomes (B).

Abbreviations: $\mathrm{C}$, coffee; NP, nanoparticle. 
biocompatible macromolecules are of enormous importance for nanoparticle synthesis. ${ }^{12,32,33}$ Although these green methods yield metal nanoparticles, the sizes, the shapes, the dispersity grades of the obtained colloids may vary from one to another green approach. In correlation with these physical and chemical properties the biological activity of the produced particles may differ significantly as well. Even though the biological entities used for the nanomaterial synthesis are composed of similar or of the same active ingredients, very often the effects of the obtained nanoparticles especially on living cells might fluctuate substantially. Therefore, in this present work our goal was not to prove that GT or C extracts are more advantageous for AgNP synthesis than another green materials, but rather to emphasize that the biological activity or the toxicity profiles of the prepared nanomaterials might differ considerably depending on the applied green extract. Thus, we have not only produced two types (C-AgNP and GT-AgNPs) of AgNPs using green technologies based on two extracts with similar active components, and characterized the physicochemical properties of the particles, but as well compared them in an extensive screening against various microbes and mammalian cells to feature major differences between their biological efficiencies and toxicities.

Both C and GT extracts have been successfully applied in our environmentally benign production of AgNPs. Generally, natural extracts contain a large number of biologically active components, many of which can be responsible for the reduction of silver ions as well as for the stabilization of the obtained nanoparticles. It would be a major challenge to identify all the chemical constituents of either extract; however, according to previous studies the key phytochemicals responsible for converting silver ions into AgNPs thus functioning in this green synthesis approach as bioreductants could be phenolics, coumarins, ubiquinones, terpenoids, glycosides, alkaloids, and tannins. Furthermore, peptides and proteins in C and GT extracts with their cysteine amino acid side chains could also act to favor silver ion reduction and nanoparticle production. ${ }^{25,34,35}$ Moreover, the carbonyl groups of proteins have strong binding ability to metal nanoparticles and thus proteins can form a coating layer on the surface of the AgNPs, thereby preventing agglomeration and enhancing the stability of the synthesized nanoparticles in aqueous media..$^{25,34-36}$

In this study, we found that both AgNPs proved to be effective in the examined concentrations against nearly all the tested microbes; however, GT-AgNPs performed always markedly better in toxicity and antimicrobial screens than $\mathrm{C}-\mathrm{AgNP}$ counterparts. It is also noteworthy that apart from their antimicrobial activity, GT-AgNPs were also highly toxic against mammalian cells, which limits their potential applications. On the contrary, C-AgNPs exhibited an extensive inhibitory action on $\mathrm{Cr}$. neoformans as well as on E. coli; however, these particles were biocompatible with the tested HeLa and NIH/3T3 cells, showing no mammalian cytotoxicity.

Apart from antibacterial and antifungal features, we tried to assess the antiviral activity of the produced AgNPs. As there is no ideal experimental model for screening the antiviral propensity of any nanomaterial produced via conventional or green synthesis approaches, we have tested GT-AgNPs and C-AgNPs on S. cerevisiae dsRNA viruses. There are some major advantages of using this model system, namely that the host strain $S$. cerevisiae itself is less susceptible to C-AgNP or GT-AgNP treatments than other yeast strains. Furthermore, the possible influence of the AgNPs on viral replication can be detected easily by loss of killer phenotype of the host strain and by checking the presence of the viral genome. We detected killer-curing capacity of both AgNPs, as in some cases the loss of killer phenotype was observed after AgNP exposures. However, as viral genomes could be identified in the nonkiller strains as well after multiple cycles of cultivation, the alteration in phenotype is not the consequence of the eradication of the viruses themselves.

Generally, it is accepted that smaller nanoparticles possess higher toxicity due to their larger relative surface area, which enables the more intensive release of reactive silver ions. The mechanism of AgNP action is in fact driven mainly by these ions, leading to the generation of reactive oxygen species finally causing cell death. ${ }^{37,38}$ Therefore, the less silver ions are released, the weaker will be the AgNPinduced cellular toxicity. Surprisingly, in every biological test we performed, bigger sized GT-AgNPs resulted to be more effective than smaller C-AgNPs. This phenomenon might be the direct consequence of the thick matrix, where C-AgNPs seem to be completely embedded (Figure 1A). This moiety of multicomponent matrix on the surface and in the environment of C-AgNPs supposedly attenuates or retards the release of silver ions from the nanoparticle surface; thus, this inhibitory effect on nanoparticle dissolution results in reduced cytotoxicity. In fact, ICP-MS measurements verified that $\sim 3.5$ times more silver ions can be released from GT-AgNPs than from C-AgNPs. Therefore, we concluded that the lower Ag-ion-releasing capability of C-AgNPs is responsible for the weaker biological activity. However, it has to be emphasized that C-AgNPs were also effective against 
microbes without being cytotoxic, which renders C-AgNPs as attractive potential candidates for further applications.

These results clearly show that the various green materials used for stabilization and for the reduction of metal ions have a defining role in determining or fine-tuning the biological activity of the obtained nanoparticles. Therefore, more careful considerations in selecting green substances for the nanomaterial production and thorough screening regimens of the obtained nanomaterials are required to estimate their efficiencies.

\section{Conclusion}

Nanomaterial industry produces enormous amounts of materials for electronics, optics, diagnostics, and therapy. Therefore, a shift to green synthesis approaches for nanomaterial production using renewable resources, eco-friendly, and biocompatible substances directly from nature, avoiding toxic chemicals and leaving only innocuous waste materials behind would support sustainable development and reduce the ecological footprint of this industry. It should be noted, however, that since the given green material or entity used for the nanomaterial synthesis can largely define the physical, chemical, and biological characteristics of the obtained nanomaterial, a comprehensive screen of the products should be carried out prior their applications to delineate their behavior in the presence of living systems.

\section{Disclosure}

The authors report no conflicts of interest in this work.

\section{References}

1. Ge L, Li Q, Wang M, Ouyang J, Li X, Xing MM. Nanosilver particles in medical applications: synthesis, performance, and toxicity. Int $J$ Nanomedicine. 2014;9:2399-2407.

2. Huang HT, Yang Y. Preparation of silver nanoparticles in inorganic clay suspensions. Compos Sci Technol. 2008;68(14):2948-2953.

3. Jung JH, Oh HC, Noh HS, Ji JH, Kim SS. Metal nanoparticle generation using a small ceramic heater with a local heating area. J Aerosol Sci. 2006; 37(12):1662-1670.

4. Socol Y, Abramson O, Gedanken A, Meshorer Y, Berenstein L, Zaban A. Suspensive electrode formation in pulsed sonoelectrochemical synthesis of silver nanoparticles. Langmuir. 2002;18(12):4736-4740.

5. Nadagouda MN, Speth TF, Varma RS. Microwave-assisted green synthesis of silver nanostructures. Accounts Chem Res. 2011;44(7):469-478.

6. Wiley B, Sun YG, Mayers B, Xia YN. Shape-controlled synthesis of metal nanostructures: the case of silver. Chem-Eur J. 2005;11(2):454-463.

7. Zhang YH, Chen F, Zhuang JH, et al. Synthesis of silver nanoparticles via electrochemical reduction on compact zeolite film modified electrodes. Chem Commun. 2002;23:2814-2815.

8. Janardhanan R, Karuppaiah M, Hebalkar N, Rao TN. Synthesis and surface chemistry of nano silver particles. Polyhedron. 2009;28(12): $2522-2530$

9. Kharissova OV, Dias HV, Kharisov BI, Perez BO, Perez VM. The greener synthesis of nanoparticles. Trends Biotechnol. 2013;31(4): 240-248.
10. Sharma VK, Yngard RA, Lin Y. Silver nanoparticles: green synthesis and their antimicrobial activities. Adv Colloid Interface Sci. 2009;145(1-2): 83-96.

11. Kozma G, Ronavari A, Konya Z, Kukovecz A. Environmentally benign synthesis methods of zero-valent iron nanoparticles. ACS Sustain Chem Eng. 2016;4(1):291-297.

12. Narayanan KB, Sakthivel N. Green synthesis of biogenic metal nanoparticles by terrestrial and aquatic phototrophic and heterotrophic eukaryotes and biocompatible agents. Adv Colloid Interface Sci. 2011; 169(2):59-79.

13. Joerger R, Klaus T, Granqvist CG. Biologically produced silvercarbon composite materials for optically functional thin-film coatings. Adv Mater. 2000;12(6):407-409.

14. Moghaddam AB, Namvar F, Moniri M, Tahir PM, Azizi S, Mohamad R. Nanoparticles biosynthesized by fungi and yeast: a review of their preparation, properties, and medical applications. Molecules. 2015; 20(9):16540-16565.

15. Mittal AK, Chisti Y, Banerjee UC. Synthesis of metallic nanoparticles using plant extracts. Biotechnol Adv. 2013;31(2):346-356.

16. Rao NH, N L, Pammi SV, Kollu P, S G, P L. Green synthesis of silver nanoparticles using methanolic root extracts of Diospyros paniculata and their antimicrobial activities. Mater Sci Eng C Mater Biol Appl. 2016; 62:553-557.

17. Ajitha B, Reddy YA, Reddy PS. Green synthesis and characterization of silver nanoparticles using Lantana camara leaf extract. Mater Sci Eng C Mater Biol Appl. 2015;49:373-381.

18. Patra S, Mukherjee S, Barui AK, Ganguly A, Sreedhar B, Patra CR. Green synthesis, characterization of gold and silver nanoparticles and their potential application for cancer therapeutics. Mater Sci Eng C Mater Biol Appl. 2015;53:298-309.

19. Krishnaraj C, Jagan EG, Rajasekar S, Selvakumar P, Kalaichelvan PT, Mohan N. Synthesis of silver nanoparticles using Acalypha indica leaf extracts and its antibacterial activity against water borne pathogens. Colloid Surf B Biointerface. 2010;76(1):50-56.

20. Shankar SS, Rai A, Ahmad A, Sastry M. Rapid synthesis of Au, Ag, and bimetallic Au core-Ag shell nanoparticles using Neem (Azadirachta indica) leaf broth. J Colloid Interface Sci. 2004;275(2):496-502.

21. Chandran SP, Chaudhary M, Pasricha R, Ahmad A, Sastry M. Synthesis of gold nanotriangles and silver nanoparticles using Aloe vera plant extract. Biotechnol Progr. 2006;22(2):577-583.

22. Prathna TC, Chandrasekaran N, Raichur AM, Mukherjee A. Biomimetic synthesis of silver nanoparticles by Citrus limon (lemon) aqueous extract and theoretical prediction of particle size. Colloid Surf B Biointerface. 2011;82(1):152-159.

23. LiSK, Shen YH, Xie AJ, et al. Green synthesis of silver nanoparticles using Capsicum annuum L. extract. Green Chem. 2007;9(8):852-858.

24. Ahmed S, Ahmad M, Swami BL, Ikram S. A review on plants extract mediated synthesis of silver nanoparticles for antimicrobial applications: A green expertise. $J$ Adv Res. 2016;7(1):17-28.

25. Dhand V, Soumya L, Bharadwaj S, Chakra S, Bhatt D, Sreedhar B. Green synthesis of silver nanoparticles using Coffea arabica seed extract and its antibacterial activity. Mater Sci Eng C Mater Biol Appl. 2016; 58:36-43.

26. Loo YY, Chieng BW, Nishibuchi M, Radu S. Synthesis of silver nanoparticles by using tea leaf extract from Camellia sinensis. Int $J$ Nanomedicine. 2012;7:4263-4267.

27. Begum NA, Mondal S, Basu S, Laskar RA, Mandal D. Biogenic synthesis of $\mathrm{Au}$ and $\mathrm{Ag}$ nanoparticles using aqueous solutions of Black Tea leaf extracts. Colloid Surf B Biointerface. 2009;71(1):113-118.

28. Ronavari A, Kovacs D, Vagvolgyi C, Konya Z, Kiricsi M, Pfeiffer I. Ion exchange defines the biological activity of titanate nanotubes. J Basic Microbiol. 2016;56(5):557-565.

29. Magliani W, Conti S, Gerloni M, Bertolotti D, Polonelli L. Yeast killer systems. Clin Microbiol Rev. 1997;10(3):369-400.

30. Sun Y, Xia Y. Shape-controlled synthesis of gold and silver nanoparticles. Science. 2002;298(5601):2176-2179. 
31. Szymczycha-Madeja A, Welna M, Zyrnicki W. Multi-element analysis, bioavailability and fractionation of herbal tea products. $J$ Brazil Chem Soc. 2013;24(5):777-787.

32. Bhattacharya D, Gupta RK. Nanotechnology and potential of microorganisms. Crit Rev Biotechnol. 2005;25(4):199-204.

33. Poulose S, Panda T, Nair PP, Theodore T. Biosynthesis of silver nanoparticles. J Nanosci Nanotechnol. 2014;14(2):2038-2049.

34. Loo YY, Chieng BW, Nishibuchi M, Radu S. Synthesis of silver nanoparticles by using tea leaf extract from Camellia sinensis. Int $J$ Nanomed. 2012;7:4263-4267.

35. Baharara J, Namvar F, Ramezani T, Mousavi M, Mohamad R. Silver nanoparticles biosynthesized using Achillea biebersteinii flower extract: apoptosis induction in MCF-7 cells via caspase activation and regulation of Bax and Bcl-2 gene expression. Molecules. 2015;20(2): 2693-2706.
36. Vivek R, Thangam R, Muthuchelian K, Gunasekaran P, Kaveri K, Kannan S. Green biosynthesis of silver nanoparticles from Annona squamosa leaf extract and its in vitro cytotoxic effect on MCF-7 cells. Process Biochem. 2012;47(12):2405-2410.

37. Kovacs D, Igaz N, Keskeny C, et al. Silver nanoparticles defeat p53positive and p53-negative osteosarcoma cells by triggering mitochondrial stress and apoptosis. Sci Rep. 2016;6:27902.

38. Igaz N, Kovacs D, Razga Z, Konya Z, Boros IM, Kiricsi M. Modulating chromatin structure and DNA accessibility by deacetylase inhibition enhances the anti-cancer activity of silver nanoparticles. Colloids Surf B Biointerfaces. 2016;146:670-677. 


\section{Supplementary materials}

A

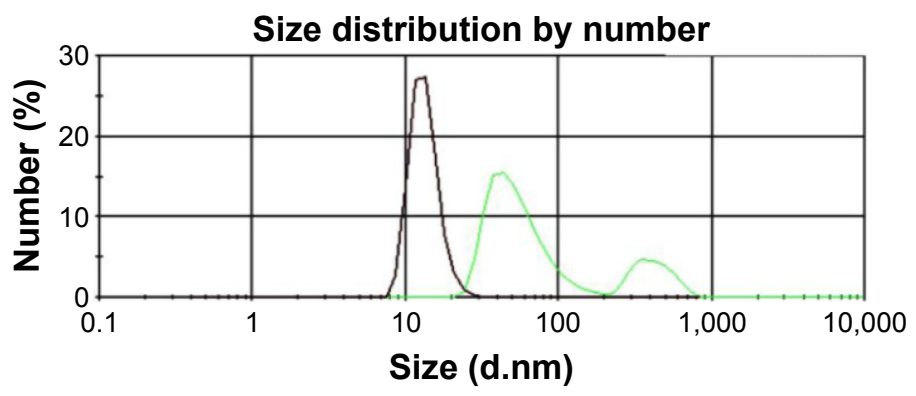

B

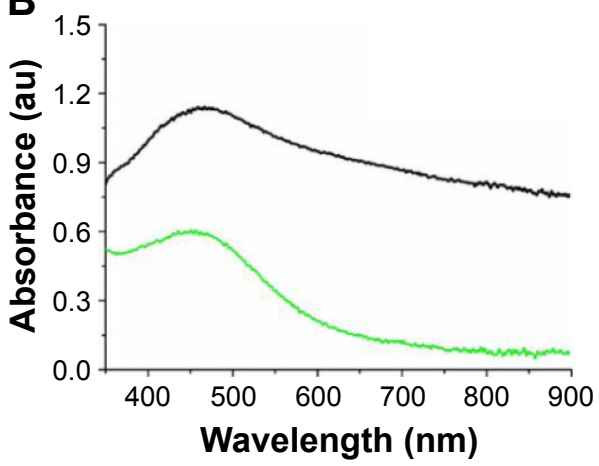

C-AgNP - GT-AgNP

Figure SI DLS (A) and UV-VIS spectra (B) of GT-AgNPs and C-AgNPs 3 months after synthesis. Abbreviations: C, coffee; GT, green tea; NP, nanoparticle.

Table SI Inhibition zones in the presence of AgNPs

\begin{tabular}{lll}
\hline Microorganisms & C-AgNP inhibition zone $\mathbf{( m m})$ & GT-AgNP inhibition zone $(\mathbf{m m})$ \\
\hline Bacillus cereus var. mycoides & $1.38 \pm 0.24$ & $1.63 \pm 0.13$ \\
Micrococcus luteus & $3.00 \pm 0.00$ & $4.00 \pm 0.00$ \\
Escherichia coli & $1.75 \pm 0.14$ & $2.25 \pm 0.14$ \\
Pseudomonas aeruginosa & $<1.00$ & $1.00 \pm 0.00$ \\
Candida albicans & $4.00 \pm 0.00$ & $4.00 \pm 0.57$ \\
Cryptococcus neoformans & $4.50 \pm 0.20$ & $4.75 \pm 0.14$ \\
Candida parapsilosis & $<1.00$ & $1.66 \pm 0.57$ \\
Saccharomyces cerevisiae & 0.00 & 0.00
\end{tabular}

Notes: The concentration of C-AgNP and GT-AgNP was $17.5 \mathrm{mg} / \mathrm{mL}$. Number of samples $(n=4)$. Abbreviations: C, coffee; GT, green tea; NP, nanoparticle.
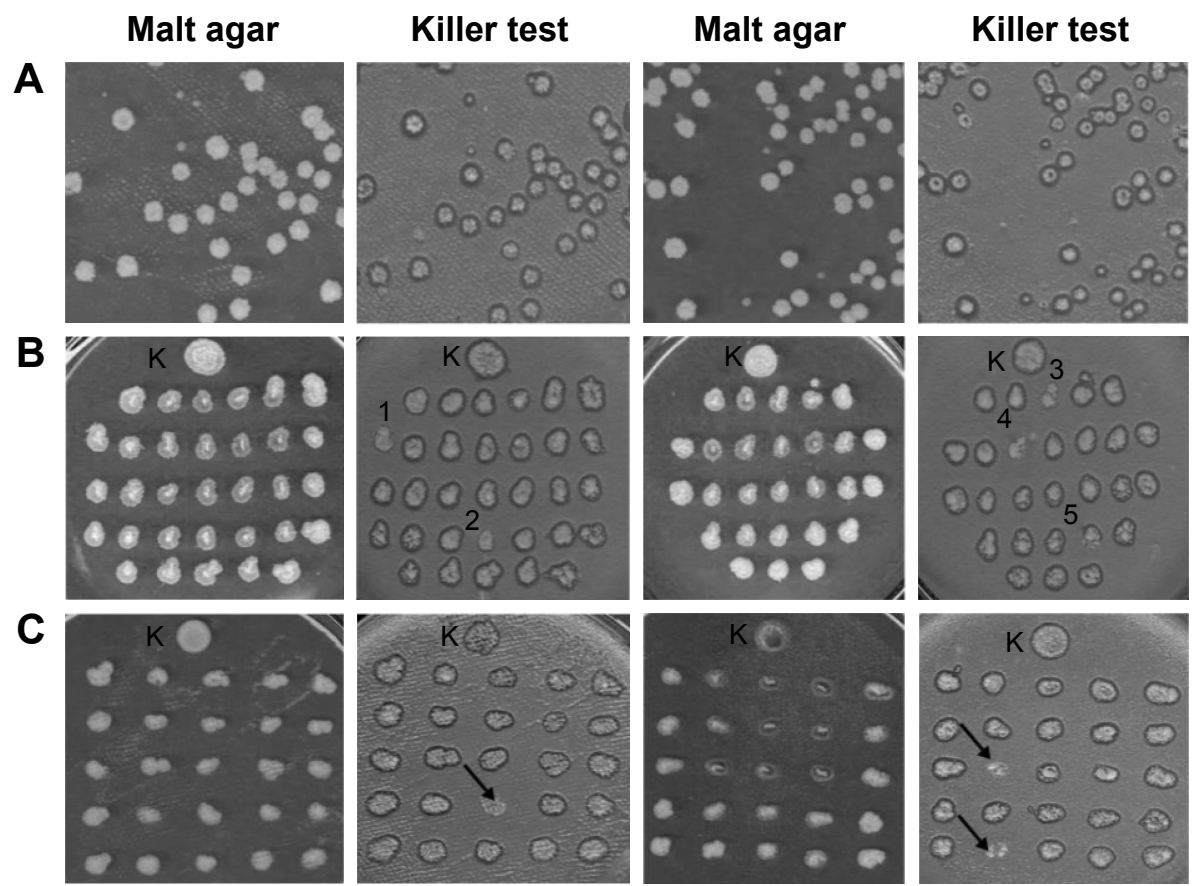

Figure S2 Killer activity of untreated (A), C-AgNP-treated (B), and GT-AgNP-treated Saccharomyces cerevisiae SZMC 20733 cells (C). Numbers (I-5) indicate the colonies of C-AgNP-treated cells where no killer phenotype was observed, whereas GT-AgNP-treated colonies with lost killer activity are indicated with black arrows. Abbreviations: C, coffee; GT, green tea; NP, nanoparticle. 
International Journal of Nanomedicine

Dovepress

\section{Publish your work in this journal}

The International Journal of Nanomedicine is an international, peerreviewed journal focusing on the application of nanotechnology in diagnostics, therapeutics, and drug delivery systems throughou the biomedical field. This journal is indexed on PubMed Central, MedLine, CAS, SciSearch ${ }^{\circledR}$, Current Contents ${ }^{\circledR} /$ Clinical Medicine,
Journal Citation Reports/Science Edition, EMBase, Scopus and the Elsevier Bibliographic databases. The manuscript management system is completely online and includes a very quick and fair peer-review system, which is all easy to use. Visit http://www.dovepress.com/ testimonials.php to read real quotes from published authors.

Submit your manuscript here: http://www.dovepress.com/international-journal-of-nanomedicine-journal 